\title{
A Fully-Lexicalized Probabilistic Model for Japanese Syntactic and Case Structure Analysis
}

\author{
Daisuke Kawahara $^{\dagger}$ and Sadao Kurohashi ${ }^{\dagger \dagger}$
}

\begin{abstract}
We present an integrated probabilistic model for Japanese syntactic and case structure analysis. Syntactic and case structures are simultaneously analyzed on the basis of wide-coverage case frames that are constructed from a huge raw corpus in an unsupervised manner. This model selects the syntactic and case structures that have the highest generative probability. We evaluate both syntactic structure and case structure. In particular, the experimental results for syntactic analysis on web sentences show that the proposed model significantly outperforms the known syntactic analyzers.
\end{abstract}

Key Words: case frames, corpus, web, syntactic analysis, case structure analysis

\section{Introduction}

In recent years, the accuracy of syntactic parsing has improved. The approaches for syntactic parsing can be divided into two types: rule-based methods (e.g., Kurohashi and Nagao 1994) and statistical methods (e.g., Kudo and Matsumoto 2002). Fundamentally, both types use information from parts of speech (POS) and conjugation of morphemes, punctuation, and function words to achieve high accuracy. For example, consider the following Japanese sentence: ${ }^{1}$

(1) bentou-wo tabete shuppatsu-shita

lunch box-ACC eat departed

((someone) ate a lunchbox and departed.)

These systems can correctly judge the head of "bentou-wo" (lunch box-ACC) as "tabete" (eat). This is because they consider the tendency that the phrase "-wo" (ACC) depends on the closest predicate in most cases. This kind of dependency constraint and preference based on POS, function words, and so forth, is described by rule-based methods and learned from a tagged corpus by statistical methods. However, neither method can deal with lexical preferences.

\footnotetext{
$\dagger$ National Institute of Information and Communications Technology

†† Graduate School of Informatics, Kyoto University

* This article has been partially revised for better understanding of overseas readers.

${ }^{1}$ In this article, we use the following abbreviations: NOM (nominative), ACC (accusative), DAT (dative), LOC (locative), ABL (ablative), ALL (allative), GEN (genitive), and TOP (topic marker).
} 
(2)
a. bentou-wo
shuppatsu-suru mae-ni tabeta

lunch box-ACC depart

before ate

((someone) ate a lunchbox before departing.)

b. bentou-wa tabete shuppatsu-shita

lunch box-TOP eat departed

(As for the lunchbox, (someone) ate (it) and departed.)

In example (2a), "bentou-wo" (lunch box-ACC) is handled in the same way as example (1), and the head of "bentou-wo" is incorrectly judged as "shuppatsu-suru" (depart). In example (2b), the head of "bentou-wa" (lunch box-TOP) is incorrectly judged as "shuppatsu-shita" (departed) because of the tendency that the topical phrase "-wa" (TOP) is likely to depend on a distant phrase, such as at the end of the sentence. To make these cases correct, it is necessary to learn lexical preferences such as "bentou-wo taberu" (eat a lunch box). Although statistical methods consider lexical information as features in many cases, it is difficult or almost impossible to learn lexical preferences from a training corpus comprising tens of thousands of sentences, because of the data sparseness problem.

Furthermore, even if such lexical preferences are sufficiently learned, it is difficult to analyze the following sentence correctly:

(3) Taro-ga tabeta Hanako-no bentou

Taro-NOM ate Hanako-GEN lunch box

(Hanako's lunch box that Taro ate)

If the lexical preferences of "bentou-wo taberu" (eat a lunch box) and "Hanako-ga taberu" (Hanako eats) are learned, it is not possible to determine the head of "tabeta" (ate) from such knowledge. To analyze this sentence correctly, it is necessary to recognize that "tabeta" has a "ga" (NOM) argument of "Taro" and a "wo" (ACC) argument of "bentou" (lunch box). In this way, it is important for the improvement of syntactic analysis to recognize predicate-argument structures based on lexical preferences.

To perform case structure analysis in practice, which is the task of recognizing predicateargument structures, the requisites are as large case frames as possible, which represent the relations between a predicate and its arguments. For such case frames, we can use large-scale case frames compiled from a large web corpus (Kawahara and Kurohashi 2006). We propose a probabilistic model for joint syntactic and case structure analysis on the basis of these case frames. This model performs probabilistic case structure analysis in a generative manner, and 
selects the syntactic structure that has the highest probability of case structure.

There have been several approaches that explicitly consider lexical preferences. Shirai, Inui, Tokunaga, and Tanaka (1998) and Fujio and Matsumoto (1998) used probabilities for word cooccurrences estimated from hundreds of thousands or millions of sentences. The work most closely related to our model is the syntactic analysis method of (Abekawa and Okumura 2006). They proposed a method for syntactic analysis, based on relations between arguments of a predicate and co-occurrence relations between a predicate and its arguments. These two types of relations were gathered from a 30-year volume of newspaper articles, and these probabilities were estimated using PLSI. They applied probabilistic re-ranking to n-best syntactic trees output by an existing parser, and selected the tree with the highest probability. This method introduced latent semantic classes based on PLSI, and estimated probabilities from a mid-scale corpus.

Our work is different from these previous approaches in the following aspects.

- We use case frames that are classified explicitly, according to predicate meanings and usages. We can perform precise case structure analysis by selecting an appropriate case frame and disambiguating the semantic ambiguity of predicates.

- We do not generalize corpus instances, but use instances in case frames themselves, extracted from a very large corpus.

- We define a generative model for syntactic and case structure analysis. It is different from the re-ranking model based on an n-best list (Abekawa and Okumura 2006).

\section{Automatically Constructed Case Frames}

We employ automatically compiled case frames (Kawahara and Kurohashi 2006). This section outlines the method for compiling case frames.

It is desirable to cover various linguistic expressions with case frames, which are an important part of humans' common sense. To build such case frames, we gradually extract reliable information from a large-scale corpus.

A large corpus is first automatically parsed, and initial case frames are constructed from modifier-head examples in the resulting parses. The biggest problem in automatic case frame compilation is predicate usage ambiguities. That is, a predicate may have multiple usages (or senses), and case patterns and filled instances are different for each usage. For example, the following two sentences have the same predicate but different usages. ${ }^{2}$

${ }^{2}$ Although the English translations of the predicate are different, the same predicate is used in Japanese. 
a. torakku-ni nimotsu-wo tsumu

truck-DAT baggage-ACC load

((someone) loads baggage onto a truck.)

b. keiken-wo tsumu

experience-ACC accumulate

((someone) accumulates experience.)

To divide case frames according to their usages, we make a couple of a predicate and its closest argument to be a unit of case frame compilation. Our units for case frames are "nimotsuwo tsumu" (load baggage) and "keiken-wo tsumu" (accumulate experience) for the predicate "tsumu." Furthermore, we apply clustering to these initial case frames to merge very similar expressions such as "nimotsu-wo tsumu" (load baggage) and "busshi-wo tsumu" (load supply).

The above first step of case frame compilation uses syntactic parsing, and basically collects arguments with case-marking postpositions. Therefore, the resulting case frames cannot handle complex expressions, such as double nominative constructions, non-gapping relations, and case alternation. To cope with this problem, we analyze the corpus again using the acquired case frames, and provide the case frames with new information. This new information includes knowledge about topic-marked phrases (phrases with "wa" and " $m o$ " postpositions) and clausal modifiees that cannot be handled by the first step of case frame compilation.

(5) kono kuruma-wa enjin-ga yoi this car-TOP engine-NOM good

(This car's engine is good.)

For example, syntactic analysis cannot interpret "kuruma-wa" (car-TOP). Case analysis can interpret "kuruma-wa" as an outer nominative because the case frame " $\{$ enjin $\}:$ ga yoi" (\{engine $\}$ : NOM is good) does not have case slots other than "ga" (NOM). That is, this process says that this case frame can take a double nominative construction.

(6) sono mondai-wa kare-ga toshokan-de shirabete-iru that problem-TOP he-NOM library-LOC investigate

(As for that problem, he is investigating (it) in a library.)

Since "mondai-wa" (problem-TOP) in this sentence is similar to the instances in the " $w o "$ (ACC) case slot of the case frame " $\{$ mondai, kadai\}:wo $\{$ toshokan $\}:$ de shiraberu" (\{problem, issue $\}: A C C$ library\}:LOC investigate), it is judged as an instance of "wo" (ACC) case and this process does not produce new information. Similarly, clausal modifiees are not interpreted in 
syntactic analysis but in case analysis by examining the similarity between a clausal modifiee and instances in case slots ("ga," "wo," etc.) of case frames. For example, since "menkyo" (license) in the expression "gyoumu-wo itonamu menkyo" (the license to carry on business) is not similar to any instance in the case frame " $\{$ ginkou, kaisha $\}:$ ga $\{$ gyoumu, bijinesu $\}$ :wo itonamu" ( $\{$ bank, company $\}$ NOM \{business, work $\}: A C C$ carry on), it is considered to have a non-gapping relation and is added to the case frame as new information.

Using this gradual procedure, we compiled case frames from the web corpus (Kawahara and Kurohashi 2006). The case frames were obtained from approximately 500 million sentences extracted from the web. This process was conducted using a computer grid comprising approximately 350 CPUs, and it took approximately a week. The resultant case frames consisted of 90,000 predicates. Table 1 shows some examples of the resulting case frames.

Table 1 Case frame examples (Examples are written only in English because of space limitation. The number following each example is its frequency.)

\begin{tabular}{|c|c|c|}
\hline & $\mathrm{CS}$ & examples \\
\hline $\begin{array}{l}\text { yaku (1) } \\
\text { (broil) }\end{array}$ & $\begin{array}{l}g a \\
w o \\
d e\end{array}$ & $\begin{array}{l}\text { I:18, person: } 15 \text {, craftsman: } 10, \cdots \\
\text { bread:2484, meat: } 1521 \text {, cake: } 1283, \cdots \\
\text { oven: } 1630 \text {, frying pan: } 1311 \text {, toaster: } 668, \cdots\end{array}$ \\
\hline $\begin{array}{l}\text { yaku (2) } \\
\text { (have difficulty) }\end{array}$ & $\begin{array}{l}g a \\
w o \\
n i\end{array}$ & $\begin{array}{l}\text { teacher: } 3 \text {, government:3, person: } 3, \cdots \\
\text { fingers: } 2950 \\
\text { attack: } 18, \text { action: } 15, \text { son: } 15, \cdots\end{array}$ \\
\hline $\begin{array}{l}\text { yaku (3) } \\
\text { (burn) }\end{array}$ & $\begin{array}{l}g a \\
w o \\
n i\end{array}$ & $\begin{array}{l}\text { maker:1, distributor: } 1 \\
\text { data:178, file: } 107 \text {, copy:9, } \cdots \\
\text { R:1583, CD: } 664 \text {, CDR:3, } \cdots\end{array}$ \\
\hline$\vdots$ & $\vdots$ & $\vdots$ \\
\hline $\begin{array}{l}\text { oyogu (1) } \\
\text { (swim) }\end{array}$ & $\begin{array}{l}g a \\
w o \\
d e\end{array}$ & $\begin{array}{l}\text { dolphin: } 142 \text {, tutee:50, fish: } 28, \cdots \\
\text { sea:1188, underwater:281, undersea:101, } \cdots \\
\text { crawl:86, breaststroke:49, stroke:24, } \cdots\end{array}$ \\
\hline$\vdots$ & $\vdots$ & $\vdots$ \\
\hline $\begin{array}{l}\text { migaku (1) } \\
\text { (brush) }\end{array}$ & $\begin{array}{l}g a \\
w o \\
d e\end{array}$ & $\begin{array}{l}\text { I:4, man: } 4 \text {, person: } 4, \cdots \\
\text { tooth: } 5959 \text {, molar: } 27, \text { foretooth: } 12 \\
\text { brush: } 38, \text { toilet: } 15, \text { salt: } 13, \cdots\end{array}$ \\
\hline$\vdots$ & : & $\vdots$ \\
\hline $\begin{array}{l}\text { rokuga }(1) \\
\text { (record) }\end{array}$ & $\begin{array}{l}g a \\
w o \\
n i\end{array}$ & $\begin{array}{l}\text { husband: } 4 \text {, sister: } 2 \text {, acquaintance: } 2, \cdots \\
\text { program: } 1435 \text {, broadcast:521, special program: } 26, \cdots \\
\text { video: } 3753 \text {, disc: } 256, \cdots\end{array}$ \\
\hline$\vdots$ & $\vdots$ & $\vdots$ \\
\hline
\end{tabular}




\section{Integrated Probabilistic Model for Syntactic and Case Structure Analysis}

The proposed method gives a probability to each possible syntactic structure $T$ and case structure $L$ of input sentence $S$, and outputs the syntactic and case structure that have the highest probability. That is, the system selects the syntactic structure $T_{\text {best }}$ and the case structure $L_{\text {best }}$ that maximize the probability $P(T, L \mid S)$ :

$$
\begin{aligned}
\left(T_{\text {best }}, L_{\text {best }}\right) & =\underset{(T, L)}{\operatorname{argmax}} P(T, L \mid S) \\
& =\underset{(T, L)}{\operatorname{argmax}} \frac{P(T, L, S)}{P(S)} \\
& =\underset{(T, L)}{\operatorname{argmax}} P(T, L, S) .
\end{aligned}
$$

The last equation is derived because $P(S)$ is constant.

\subsection{Generative Model for Syntactic and Case Structure Analysis}

We propose a generative probabilistic model based on dependency formalism. This model considers a clause as a unit of generation, and generates the input sentence from the end of the sentence in turn. $P(T, L, S)$ is defined as the product of a probability for generating a clause $c_{i}$ as follows:

$$
P(T, L, S)=\prod_{c_{i} \in S} P\left(c_{i} \mid b_{h}\right),
$$

where $b_{h}$ is $c_{i}$ 's modifying bunsetsu. ${ }^{3}$ The main clause $c_{n}$ at the end of a sentence does not have a modifying head, but we handle it by assuming $b_{h_{n}}=$ EOS (End Of Sentence).

Although most previous studies defined the generative probability of a sentence as the product of a dependency probability between two bunsetsus, we use a generative model of a clause, which is a predicate and its arguments, in equation (2). By virtue of this, our model can determine dependencies by considering multiple arguments and correctly analyze sentences, such as example (3).

For example, consider the sentence in Figure 1. There are two possible dependency structures, and for each structure, the product of probabilities indicated below the tree is calculated. Finally, the model chooses the highest-probability structure (in this case, the left one).

\footnotetext{
${ }^{3}$ In Japanese, bunsetsu is a basic unit of dependency, consisting of one or more content words and the following zero or more function words. It corresponds to a base phrase in English and "eojeol" in Korean.
} 


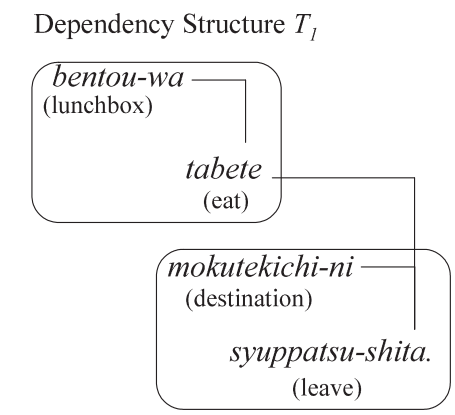

P(mokutekichi-ni syuppatsu-shita.|EOS) $\stackrel{\stackrel{\times}{\times} \text { (bentou-wa tabete|syuppatsu-shita.) }}{ }$

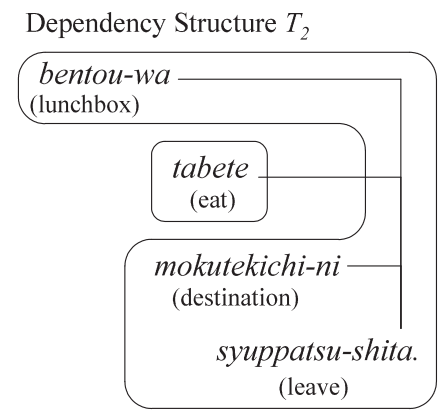

P(bentou-wa mokutekichi-ni syuppatsu-shita.|EOS)

$\stackrel{\stackrel{\times}{\times} \text { (tabete|syuppatsu-shita.) }}{ }$

Fig. 1 Example of Probability Calculation

$c_{i}$ is decomposed into its predicate type $f_{i}$ (including the predicate's inflection) and the rest case structure $C S_{i}$. This means that the predicate included in $C S_{i}$ is lemmatized. Bunsetsu $b_{h}$ is also decomposed into content part $w_{h}$ and type $f_{h}$.

$$
\begin{aligned}
P\left(c_{i} \mid b_{h}\right) & =P\left(C S_{i}, f_{i} \mid w_{h}, f_{h}\right) \\
& =P\left(C S_{i} \mid f_{i}, w_{h}, f_{h}\right) P\left(f_{i} \mid w_{h}, f_{h}\right) \\
& \approx P\left(C S_{i} \mid f_{i}, w_{h}\right) P\left(f_{i} \mid f_{h}\right) .
\end{aligned}
$$

The last equation is derived because the content part in $C S_{i}$ is independent of the type of its modifying head $\left(f_{h}\right)$, and in most cases, type $f_{i}$ is independent of the content part of its modifying head $\left(w_{h}\right)$.

For example, $P$ (bentou-wa tabete|shuppatsu-shita) is calculated as follows:

$$
P(C S(\text { bentou-wa taberu }) \mid \text { te, shuppatsu-suru }) P(\text { te } \mid \text { ta. }) .
$$

We call $P\left(C S_{i} \mid f_{i}, w_{h}\right)$ the generative model for case structure and $P\left(f_{i} \mid f_{h}\right)$ the generative model for predicate type. The following two sections describe these models.

\subsection{Generative Model for Case Structure}

We propose a generative probabilistic model of case structure. This model selects a case frame that matches the input case components and makes correspondences between input case components and case slots.

A case structure $C S_{i}$ consists of a predicate $v_{i}$, a case frame $C F_{l}$ and a case assignment $C A_{k}$. Case assignment $C A_{k}$ represents correspondences between the input case components and case 


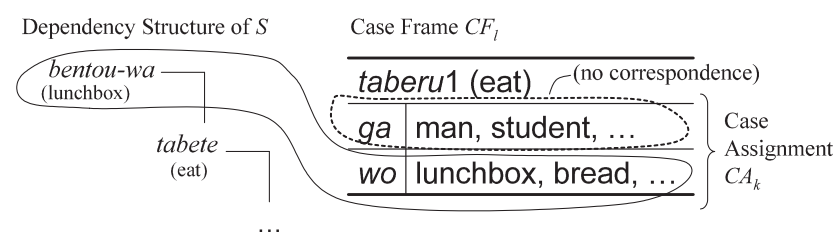

Fig. 2 Example of case assignment $C A_{k}$

slots, as shown in Figure 2. Note that there are various possibilities of case assignment in addition to that in Figure 2, such as a corresponding "bentou" (lunchbox) with "ga" case. Accordingly, the index $k$ of $C A_{k}$ ranges up to the number of possible case assignments. By splitting $C S_{i}$ into $v_{i}, C F_{l}$ and $C A_{k}, P\left(C S_{i} \mid f_{i}, w_{h}\right)$ is rewritten as follows:

$$
\begin{aligned}
P\left(C S_{i} \mid f_{i}, w_{h}\right)= & P\left(v_{i}, C F_{l}, C A_{k} \mid f_{i}, w_{h}\right) \\
= & P\left(v_{i} \mid f_{i}, w_{h}\right) \\
& \times P\left(C F_{l} \mid f_{i}, w_{h}, v_{i}\right) \\
& \times P\left(C A_{k} \mid f_{i}, w_{h}, v_{i}, C F_{l}\right) \\
\approx & P\left(v_{i} \mid w_{h}\right) \\
& \times P\left(C F_{l} \mid v_{i}\right) \\
& \times P\left(C A_{k} \mid C F_{l}, f_{i}\right) .
\end{aligned}
$$

The above approximation is given because it is natural to consider that the predicate $v_{i}$ depends on its modifying head $w_{h}$, that the case frame $C F_{l}$ only depends on the predicate $v_{i}$, and that the case assignment $C A_{k}$ depends on the case frame $C F_{l}$ and the predicate type $f_{i}$.

The probabilities $P\left(v_{i} \mid w_{h}\right)$ and $P\left(C F_{l} \mid v_{i}\right)$ are estimated from the case structure analysis results of a large raw corpus. The remainder of this section illustrates $P\left(C A_{k} \mid C F_{l}, f_{i}\right)$ in detail.

\subsubsection{Generative Probability of Case Assignment}

Let us consider case assignment $C A_{k}$ for each case slot $s_{j}$ in case frame $C F_{l} . P\left(C A_{k} \mid C F_{l}, f_{i}\right)$ can be decomposed into the following product depending on whether a case slot $s_{j}$ is filled with an input case component (content part $n_{j}$ and type $f_{j}$ ) or vacant:

$$
\begin{aligned}
P\left(C A_{k} \mid C F_{l}, f_{i}\right)= & \prod_{s_{j}: A\left(s_{j}\right)=1} P\left(A\left(s_{j}\right)=1, n_{j}, f_{j} \mid C F_{l}, f_{i}, s_{j}\right) \\
& \times \prod_{s_{j}: A\left(s_{j}\right)=0} P\left(A\left(s_{j}\right)=0 \mid C F_{l}, f_{i}, s_{j}\right)
\end{aligned}
$$




$$
\begin{aligned}
& =\prod_{s_{j}: A\left(s_{j}\right)=1}\left\{P\left(A\left(s_{j}\right)=1 \mid C F_{l}, f_{i}, s_{j}\right) \times P\left(n_{j}, f_{j} \mid C F_{l}, f_{i}, A\left(s_{j}\right)=1, s_{j}\right)\right\} \\
& \quad \times \prod_{s_{j}: A\left(s_{j}\right)=0} P\left(A\left(s_{j}\right)=0 \mid C F_{l}, f_{i}, s_{j}\right),
\end{aligned}
$$

where the function $A\left(s_{j}\right)$ returns 1 if a case slot $s_{j}$ is filled with an input case component; otherwise 0 .

$P\left(A\left(s_{j}\right)=1 \mid C F_{l}, f_{i}, s_{j}\right)$ and $P\left(A\left(s_{j}\right)=0 \mid C F_{l}, f_{i}, s_{j}\right)$ in equation (5) can be rewritten as $P\left(A\left(s_{j}\right)=1 \mid C F_{l}, s_{j}\right)$ and $P\left(A\left(s_{j}\right)=0 \mid C F_{l}, s_{j}\right)$ because the evaluation of case slot assignment depends only on the case frame. We call these probabilities the generative probability of a case slot, which are estimated from the case structure analysis results of a large corpus.

Let us calculate $P\left(C S_{i} \mid f_{i}, w_{h}\right)$ by using the example in Figure 1. In the sentence, " $w a$ " is a topic marking (TOP) postposition, and hides the case marker. The generative probability of the case structure varies depending on the case slot to which the topic marked phrase is assigned. For example, when a case frame of "taberu" (eat) $C F_{\text {taberu1 }}$ with "ga" and "wo" case slots is used, $P(C S$ (bentou-wa taberu $) \mid$ te, shuppatsu-suru $)$ is calculated as follows:

$$
\begin{aligned}
& P_{1}(C S \text { (bentou-wa taberu)|te, shuppatsu-suru) } \\
& =P(\text { taberu } \mid \text { shuppatsu-suru }) \\
& \times P\left(C F_{\text {taberu1 }} \mid \text { taberu }\right) \\
& \times P\left(\text { bentou, wa } \mid C F_{\text {taberu1 }}, \text { te }, A(w o)=1, w o\right) \\
& \times P\left(A(w o)=1 \mid C F_{\text {taberu1 }}, w o\right) \\
& \times P\left(A(g a)=0 \mid C F_{\text {taberu1 }}, g a\right), \\
& P_{2}(C S \text { (bentou-wa taberu)|te, syupatsu-suru }) \\
& =P(\text { taberu } \mid \text { shuppatsu-suru }) \\
& \times P\left(C F_{\text {taberu1 }} \mid \text { taberu }\right) \\
& \times P\left(\text { bentou, wa } \mid C F_{\text {taberu1 }}, t e, A(g a)=1, g a\right) \\
& \times P\left(A(g a)=1 \mid C F_{\text {taberu1 }}, g a\right) \\
& \times P\left(A(w o)=0 \mid C F_{\text {taberu1 }}, w o\right) .
\end{aligned}
$$

Such probabilities are computed for each case frame of "taberu" (eat), and the case frame and its corresponding case assignment that have the highest probability are selected. 
We describe the generative probability of a case component $P\left(n_{j}, f_{j} \mid C F_{l}, f_{i}, A\left(s_{j}\right)=1, s_{j}\right)$ below.

\subsubsection{Generative Probability of Case Component}

We approximate the generative probability of a case component, assuming that

- a generative probability of content part $n_{j}$ is independent of that of type $f_{j}$,

- the interpretation of the surface case included in $f_{j}$ does not depend on case frames.

Considering these assumptions, the generative probability of a case component is approximated as follows:

$$
\begin{aligned}
& P\left(n_{j}, f_{j} \mid C F_{l}, f_{i}, A\left(s_{j}\right)=1, s_{j}\right) \\
& \quad \approx P\left(n_{j} \mid C F_{l}, A\left(s_{j}\right)=1, s_{j}\right) P\left(f_{j} \mid s_{j}, f_{i}\right) .
\end{aligned}
$$

$P\left(n_{j} \mid C F_{l}, A\left(s_{j}\right)=1, s_{j}\right)$ is the probability of generating a content part $n_{j}$ from a case slot $s_{j}$ in a case frame $C F_{l}$. This probability is estimated from case frames.

Let us consider $P\left(f_{j} \mid s_{j}, f_{i}\right)$ in equation (8). This is the probability of generating type $f_{j}$ of a case component that has a correspondence with the case slot $s_{j}$. Since type $f_{j}$ consists of a surface case $c_{j},{ }^{4}$ a punctuation mark (comma) $p_{j}$ and a topic marker " $w a " t_{j}, P\left(f_{j} \mid s_{j}, f_{i}\right)$ is rewritten as follows (using the chain rule):

$$
\begin{aligned}
P\left(f_{j} \mid s_{j}, f_{i}\right)= & P\left(c_{j}, t_{j}, p_{j} \mid s_{j}, f_{i}\right) \\
= & P\left(c_{j} \mid s_{j}, f_{i}\right) \\
& \times P\left(p_{j} \mid s_{j}, f_{i}, c_{j}\right) \\
& \times P\left(t_{j} \mid s_{j}, f_{i}, c_{j}, p_{j}\right) \\
\approx & P\left(c_{j} \mid s_{j}\right) \\
& \times P\left(p_{j} \mid f_{i}\right) \\
& \times P\left(t_{j} \mid f_{i}, p_{j}\right) .
\end{aligned}
$$

This approximation is given by assuming that $c_{j}$ only depends on $s_{j}, p_{j}$ only depends on $f_{j}$, and $t_{j}$ depends on $f_{j}$ and $p_{j} . P\left(c_{j} \mid s_{j}\right)$ is estimated from the Kyoto University Text Corpus (Kawahara, Kurohashi, and Hasida 2002), in which the relation between a surface case marker and a case slot is annotated by hand.

4 A surface case means a postposition sequence at the end of bunsetsu, such as "ga," "wo," "koso" and "demo." 
In Japanese, a punctuation mark and a topic marker are likely to be used when their belonging bunsetsu has a long distance dependency. By considering such tendency, $f_{i}$ can be regarded as $\left(o_{i}, u_{i}\right)$, where $o_{i}$ means whether a dependent bunsetsu gets over another head candidate before its modifying head $v_{i}$, and $u_{i}$ means a clause type of $v_{i}$. The value of $o_{i}$ is binary, and $u_{i}$ is one of the clause types described in (Kawahara and Kurohashi 1999).

$$
\begin{gathered}
P\left(p_{j} \mid f_{i}\right)=P\left(p_{j} \mid o_{i}, u_{i}\right), \\
P\left(t_{j} \mid f_{i}, p_{j}\right)=P\left(t_{j} \mid o_{i}, u_{i}, p_{j}\right) .
\end{gathered}
$$

\subsection{Generative Model for Predicate Type}

Now, consider $P\left(f_{i} \mid f_{h}\right)$ in equation (3). This is the probability of generating the predicate type of a clause $c_{i}$ that modifies $b_{h}$. This probability varies depending on the type of $b_{h}$.

When $b_{h}$ is a predicate bunsetsu, $c_{i}$ is a subordinate clause embedded in the clause of $b_{h}$. For types $f_{i}$ and $f_{h}$, it is necessary to consider punctuation marks $\left(p_{i}, p_{h}\right)$ and clause types $\left(u_{i}, u_{h}\right)$. To capture a long distance dependency indicated by punctuation marks, $o_{h}$ (whether $c_{i}$ has a possible head candidate before $b_{h}$ ) is also considered.

$$
P_{V B m o d}\left(f_{i} \mid f_{h}\right)=P_{V B m o d}\left(p_{i}, u_{i} \mid p_{h}, u_{h}, o_{h}\right) .
$$

When $b_{h}$ is a noun bunsetsu, $c_{i}$ is an embedded clause in $b_{h}$. In this case, clause types and a punctuation mark of the modifiee do not affect the probability.

$$
P_{N B m o d}\left(f_{i} \mid f_{h}\right)=P_{N B m o d}\left(p_{i} \mid o_{h}\right)
$$

\section{Experiments}

We evaluated the syntactic structure and case structure output by our model. Each parameter was estimated using maximum likelihood from the data described in Table 2. These data are not all obtainable by a single process, but acquired by applying syntactic analysis, case frame construction, and case structure analysis in turn. The process of case structure analysis in this table is a similarity-based method (Kawahara and Kurohashi 2002). The case frames were automatically constructed from the web corpus comprising approximately 500 million sentences, and the case structure analysis results were obtained from approximately six million sentences in the web corpus. 


\subsection{Experiments for Syntactic Structure}

We evaluated syntactic structures analyzed by the proposed model. Our experiments were run on 675 hand-annotated web sentences. ${ }^{5}$ These web sentences were manually annotated using the same criteria as the Kyoto University Text Corpus. The system input was tagged automatically using the JUMAN morphological analyzer (Kurohashi, Nakamura, Matsumoto, and Nagao 1994). The syntactic structures obtained were evaluated with regard to dependency accuracy - the proportion of correct dependencies out of all dependencies, except for the last one at the sentence end. ${ }^{6}$

Table 3 shows the dependency accuracy. In the table, "CaboCha" means the SVMs-based statistical syntactic parser, CaboCha; "KNP" means the rule-based syntactic parser, KNP (Kurohashi and Nagao 1994); and "proposed" represents the proposed method. All the systems are given the same outputs of morphological analysis. To compare dependency accuracy

Table 2 Data for parameter estimation

\begin{tabular}{l|l|l}
\hline \multicolumn{1}{c|}{ probability } & what is generated & \multicolumn{1}{c}{ data } \\
\hline$P\left(p_{j} \mid o_{i}, u_{j}\right)$ & punctuation mark & Kyoto University Text Corpus \\
$P\left(t_{j} \mid o_{i}, u_{i}, p_{j}\right)$ & topic marker & Kyoto University Text Corpus \\
$P\left(p_{i}, u_{i} \mid p_{h}, u_{h}, o_{h}\right)$ & predicate type & Kyoto University Text Corpus \\
$P\left(c_{j} \mid s_{j}\right)$ & surface case & Kyoto University Text Corpus \\
$P\left(v_{i} \mid w_{h}\right)$ & predicate & parsing results \\
$P\left(n_{j} \mid C F_{l}, A\left(s_{j}\right)=1, s_{j}\right)$ & words & case frames \\
$P\left(C F_{l} \mid v_{i}\right)$ & case frame & case structure analysis results \\
$P\left(A\left(s_{j}\right)=\{0,1\} \mid C F_{l}, s_{j}\right)$ & case slot & case structure analysis results \\
\hline
\end{tabular}

Table 3 Experimental results for syntactic analysis

\begin{tabular}{l|cc|cc|cc}
\hline & \multicolumn{2}{|c|}{ CaboCha } & \multicolumn{2}{c|}{ KNP } & \multicolumn{2}{c}{ proposed } \\
\hline \multicolumn{1}{c|}{ all } & $3,412 / 3,976(85.8 \%)$ & $3,447 / 3,976$ & $(86.7 \%)$ & $3,477 / 3,976$ & $(87.4 \%)$ \\
\hline $\mathrm{NB} \rightarrow \mathrm{VB}$ & $1,308 / 1,547$ & $(84.6 \%)$ & $1,310 / 1,547$ & $(84.7 \%)$ & $1,328 / 1,547$ & $(85.8 \%)$ \\
$\mathrm{TOP}$ & $233 / 298$ & $(78.2 \%)$ & $244 / 298$ & $(81.9 \%)$ & $242 / 298$ & $(81.2 \%)$ \\
others & $1,075 / 1,249$ & $(86.1 \%)$ & $1,066 / 1,249$ & $(85.3 \%)$ & $1,086 / 1,249$ & $(\underline{86.9 \%)}$ \\
$\mathrm{NB} \rightarrow \mathrm{NB}$ & $512 / 556$ & $(92.1 \%)$ & $525 / 556$ & $(94.4 \%)$ & $526 / 556$ & $(94.6 \%)$ \\
$\mathrm{VB} \rightarrow \mathrm{VB}$ & $596 / 760$ & $(78.4 \%)$ & $593 / 760$ & $(78.0 \%)$ & $601 / 760$ & $(79.1 \%)$ \\
$\mathrm{VB} \rightarrow \mathrm{NB}$ & $452 / 497$ & $(90.9 \%)$ & $453 / 497$ & $(91.1 \%)$ & $457 / 497$ & $(92.0 \%)$ \\
\hline
\end{tabular}

\footnotetext{
5 The test set is not used for case frame construction and probability estimation.

${ }^{6}$ Since Japanese is head-final, the second last bunsetsu unambiguously depends on the last bunsetsu, and the last bunsetsu has no dependency.

7 http://chasen.org/ taku/software/cabocha/ (We used the last version of CaboCha (0.36), which can take the output of the morphological analyzer JUMAN as an input.)
} 
fairly, bunsetsu segmentations for CaboCha are coincided with KNP by inputting the bunsetsu segmentations by KNP into CaboCha. The proposed method significantly outperformed the two baselines (McNemar's test; $p<0.05$ ). The dependency accuracies are classified into four types, according to the bunsetsu classes (VB: verb bunsetsu, NB: noun bunsetsu) of a dependent and its head. The "NB $\rightarrow$ VB" type is further divided into two types: "TOP" (topic-marked phrases) and "others." The type that is most related to case structure is "others" in "NB $\rightarrow$ VB." Its accuracy was improved by $1.6 \%$, and the error rate was reduced by $10.9 \%$, compared to KNP. This result indicated that the proposed method is effective in analyzing dependencies related to the case structure.

Figure 3 shows some analysis results. Sentences (1) and (2) are incorrectly analyzed by KNP, but correctly analyzed by the proposed method.

There are two major causes that led to the analysis errors.

(1)

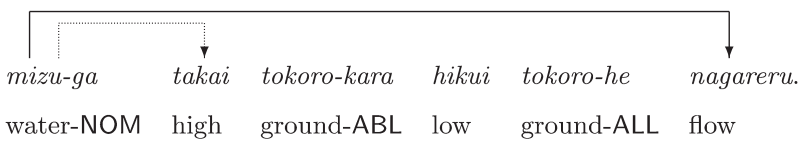

(Water flows from high ground to low ground.)

(2)

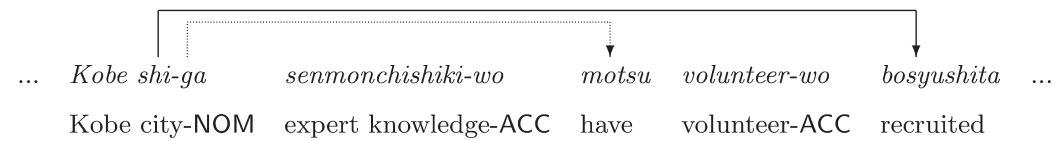

(Kobe city recruited a volunteer who has expert knowledge, ...)

(3)

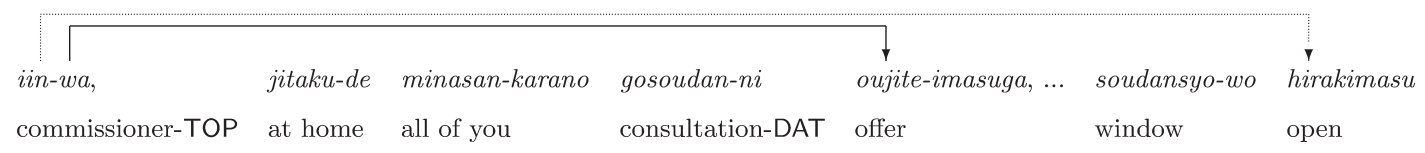

(the commissioner offers consultation to all of you at home, but opens a window ...)

(4)

\begin{tabular}{llllll}
\cline { 2 - 3 } & \multicolumn{1}{c}{ kohi-wo } & osagashi-no & kata-ni & osusume-no & saito-desu \\
yummy & coffee-ACC & looking for-GEN & people-DAT & recommendation-GEN & site
\end{tabular}

(This is a site recommended for people who are looking for yummy coffee beans.)

Fig. 3 Examples of analysis. The dotted lines represent the analysis by KNP and the solid lines represent the analysis by our proposed method. 


\subsubsection{Mismatch between the analysis results and annotation criteria}

In sentence (3) in Figure 3, KNP correctly recognized the head of "iin-wa" (commissionerTOP) as "hirakimasu" (open). However, the proposed method incorrectly judged it as "oujiteimasuga" (offer). Both analysis results can be considered to be semantically correct, but from the viewpoint of our annotation criteria, the latter is not a syntactic relation, but an ellipsis relation. To address this problem, it is necessary to simultaneously evaluate not only syntactic relations but also indirect relations, such as ellipses and anaphora.

\subsubsection{Dependency constraints}

The correct syntactic structure is not always included in the list of possible structures output by KNP. In sentence (4) in Figure 3, the correct head of "kohi-wo" (coffee-ACC) is "osagashi-no" (looking for-GEN). Since "osagashi-no" is not a possible head of "kohi-wo," our proposed method fails to detect the correct head. Although noun bunsetsus like "osagashi-no" are not usually modified, it is necessary to relax this type of constraint and search more possible structures.

\subsubsection{Linear weighting on each probability}

We proposed a generative probabilistic model, and thus cannot optimize the weight of each probability. Such optimization could be a way to improve the system performance. In the future, we plan to employ a machine learning technique for optimization.

\subsection{Experiments for Case Structure}

We applied case structure analysis to 215 web sentences, which are manually annotated with case structure, and evaluated case markers of TOP and clausal modifiees by comparing them with the gold standard in the corpus. The experimental results are shown in Table 4, in which the baseline refers to a similarity-based method (Kawahara and Kurohashi 2002). The experimental results were good compared to the baseline. It is difficult to compare the results with the previous work stated in the next section because of different experimental settings (e.g., our evaluation includes parse errors in incorrect cases).

Table 4 Experimental results for case structure analysis

\begin{tabular}{l|r|r}
\hline & baseline & \multicolumn{1}{c}{ proposed } \\
\hline TOP & $72 / 105(68.6 \%)$ & $82 / 105(78.1 \%)$ \\
clause & $107 / 155(69.0 \%)$ & $121 / 155(78.1 \%)$ \\
\hline
\end{tabular}




\subsection{Coverage of Case Frames}

To measure the coverage of case frames in the analysis process, we judged whether an argument was contained in a case frame of the modifying predicate. We evaluated dependencies that were correctly detected, and obtained a coverage of $60.7 \%$. For comparison, we conducted the same experiment using case frames compiled from a 26-year volume of newspaper articles (2.6 million sentences), obtaining a coverage of $35.1 \%$. This result indicates that the case frames from the web corpus had a high coverage.

Bikel reported that only $1.5 \%$ of bilexical dependencies in a test set were covered in a training set (Bikel 2004). Although a direct comparison is difficult because of the differences in language and resource, the coverage of our case frames is considered to be very high.

\section{Related Work}

There have been several approaches for syntactic analysis handling lexical preference on a large scale. Shirai et al. (1998) proposed a PGLR-based syntactic analysis method using large-scale lexical preference. Their system learned lexical preference from a large newspaper corpus (five years' worth of articles), such as $P$ (pie|wo, taberu), but did not deal with verb sense ambiguity. They reported an accuracy of $84.34 \%$ on 500 relatively short sentences from the Kyoto University Text Corpus.

Fujio and Matsumoto (1998) presented a syntactic analysis method based on lexical statistics. They used a probabilistic model defined by the product of a probability of having a dependency between two co-occurring words, and a distance probability. The model was trained on the EDR corpus, and performed with an accuracy of $86.89 \%$ on 10,000 sentences from the EDR corpus. ${ }^{8}$

Abekawa and Okumura (2006) proposed a method for syntactic analysis, based on the relations between arguments of a predicate and co-occurrence relations between a predicate and its arguments. Both types were gathered from a 30-year volume of newspaper articles, and these probabilities were estimated using PLSI. They applied probabilistic re-ranking to n-best syntactic trees output by an existing parser, and selected the tree with the highest probability. They achieved an accuracy of $91.21 \%$ on approximately 9,000 sentences from the Kyoto University Text Corpus, which was higher than the baseline parser by $0.26 \%$. They also integrated a method for analyzing clausal modifiees (Abekawa and Okumura 2005) into their model, and achieved an accuracy of $91.25 \%$.

\footnotetext{
8 The evaluation includes the last dependencies in the sentence end, which are always correct.
} 
There have been several machine-learning-based approaches using lexical preference as their features. Among these, Kudo and Matsumoto (2002) yielded the best performance. They proposed a chunking-based dependency analysis method using SVMs. They used two-fold cross validation on the Kyoto Text Corpus, and achieved an accuracy of $90.46 \%$. However, it is very difficult to learn sufficient lexical preferences from several tens of thousands of sentences of a hand-tagged corpus. CaboCha, which is one of the baseline methods in our experiments, is an analyzer based on this method.

\section{Conclusion}

We have described an integrated probabilistic model for syntactic and case structure analysis. This model takes advantage of lexical selectional preference of large-scale case frames, and performs syntactic and case analysis simultaneously. The experiments indicate the effectiveness of our model. In the future, by incorporating ellipsis resolution, we will develop an integrated model of syntactic, case, and ellipsis analysis.

\section{Reference}

Abekawa, T. and Okumura, M. (2005). "Corpus-Based Analysis of Japanese Relative Clause Constructions." In Proceedings of the 2nd International Joint Conference on Natural Language Processing, pp. 46-57.

Abekawa, T. and Okumura, M. (2006). "Japanese Dependency Parsing using Co-Occurrence Information and a Combination of Case Elements." In Proceedings of the 21st International Conference on Computational Linguistics and 44th Annual Meeting of the Association for Computational Linguistics, pp. 833-840.

Bikel, D. M. (2004). "Intricacies of Collins' Parsing Model." Computational Linguistics, 30 (4), pp. $479-511$.

Fujio, M. and Matsumoto, Y. (1998). "Japanese Dependency Structure Analysis Based on Lexicalized Statistics." In Proceedings of the 3rd Conference on Empirical Methods in Natural Language Processing, pp. 88-96.

Kawahara, D. and Kurohashi, S. (1999). "Corpus-based Dependency Analysis of Japanese Sentences using Verb Bunsetsu Transitivity." In Proceedings of the 5th Natural Language Processing Pacific Rim Symposium, pp. 387-391.

Kawahara, D. and Kurohashi, S. (2002). "Fertilization of Case Frame Dictionary for Robust Japanese Case Analysis." In Proceedings of the 19th International Conference on Computa- 
tional Linguistics, pp. 425-431.

Kawahara, D. and Kurohashi, S. (2006). "Case Frame Compilation from the Web using HighPerformance Computing." In Proceedings of the 5th International Conference on Language Resources and Evaluation.

Kawahara, D., Kurohashi, S., and Hasida, K. (2002). "Construction of a Japanese Relevancetagged Corpus." In Proceedings of the 3rd International Conference on Language Resources and Evaluation, pp. 2008-2013.

Kudo, T. and Matsumoto, Y. (2002). "Japanese Dependency Analysis using Cascaded Chunking." In Proceedings of the Conference on Natural Language Learning, pp. 29-35.

Kurohashi, S. and Nagao, M. (1994). "A Syntactic Analysis Method of Long Japanese Sentences Based on the Detection of Conjunctive Structures." Computational Linguistics, 20 (4), pp. 507-534.

Kurohashi, S., Nakamura, T., Matsumoto, Y., and Nagao, M. (1994). "Improvements of Japanese Morphological Analyzer JUMAN." In Proceedings of the International Workshop on Sharable Natural Language, pp. 22-28.

Shirai, K., Inui, K., Tokunaga, T., and Tanaka, H. (1998). "An Empirical Evaluation on Statistical Parsing of Japanese Sentences using Lexical Association Statistics." In Proceedings of the 3rd Conference on Empirical Methods in Natural Language Processing, pp. 80-87.

Daisuke Kawahara: Daisuke Kawahara received his B.S. and M.S. in Electronic Science and Engineering from Kyoto University in 1997 and 1999, respectively. He obtained his Ph.D. in Informatics from Kyoto University in 2005. He is currently a researcher with National Institute of Information and Communications Technology. His research interests center on natural language processing, particularly knowledge acquisition and text understanding.

Sadao Kurohashi: Sadao Kurohashi received his Ph.D. in Electrical Engineering from Kyoto University in 1994. He is currently a professor at the Graduate School of Informatics at Kyoto University. His research interests include natural language processing, knowledge acquisition/representation, and information retrieval. 